\title{
Effective Marine (spatial) Planning: A Systematic Review Of Evidence
}

\author{
Stephen Fletcher, School of Applied Sciences, Bournemouth University, UK \\ Jonathan Potts, Department of Geography, University of Portsmouth, UK \\ Chris Butler, School of Applied Sciences, Bournemouth University, UK
}

\begin{abstract}
This paper presents a meta-analysis of the peer-reviewed literature related to marine spatial planning (MSP) in order to identify and evaluate claims of effective practice. MSP is the favoured approach to the governance of marine space in many countries and in Europe is being encouraged by the European Union's 'roadmap' for maritime spatial planning and provision of funding opportunities related to marine planning (European Commission, 2008 and 2009). A total of 96 papers were reviewed, which collectively contained a total of 49 claims of effective practice. These were dominated by claims related to stakeholder involvement, which comprised $49 \%$ of the total. It was concluded that in order to be effective, MSP should draw from the best available evidence base, involve informed stakeholders, integrate with existing sectoral and spatial plans, adopt an ecosystem-based approach at an appropriate spatial and temporal scale, be implemented through a legally binding framework, and be subject to rigorous monitoring. Despite the clear consensus around these claims, the evidence supporting these claims was rarely underpinned by primary research, making the results a reflection of what is anticipated will deliver effective practice, rather than what has been proven to deliver effective practice.
\end{abstract}

Keywords: marine spatial planning, meta-analysis, effective practice

\section{Introduction}

This paper presents a meta-analysis of the peer-reviewed literature related to marine spatial planning (MSP) in order to identify and evaluate claims of effective practice. Effective practice describes those procedures, activities, or approaches that lead to effective MSP. Identification of effective practice is important as MSP is the favoured approach to the governance of marine areas in much of the world, as demonstrated by the recent European 'Roadmap' to effective maritime spatial planning' (European Union, 2008) and legislation in European member states, including Belgium, Germany, England and Wales. There is therefore a growing body of literature from which to identify claims of what might be considered effective practice in MSP. However, there are multiple interpretations of what supports effective marine planning. These mostly arise from individual examples of marine planning practice from which it is difficult to draw wider conclusions. This is partly attributable to the limited application of MSP in the international arena and partly due to a lack of critical evaluation of MSP processes where they are undertaken. This is a pressing knowledge gap as MSP is increasingly being adopted to govern national coastal and marine resources. This paper aims to this debate through a meta-analysis of the existing research literature. 
The use of meta-analysis is relatively common in medical sciences, and increasingly so in ecological and environmental sciences, but as yet is limited in marine and coastal management and related disciplines. The key benefit of the meta-analysis method is that it allows recurrent themes within the research literature to be identified and assessed within the context of the quality of its evidence base. This can allow relatively strong conclusions to be drawn where the evidence base is strong, and research gaps to be identified where evidence is weak. Strong evidence is considered to be unbiased studies that collect and evaluate primary data. In contrast, studies grounded in opinion, or with no primary data, are considered to offer relatively weak findings as the evidence underpinning their conclusions is often uncertain.

\section{Method}

A systematic review of papers related to MSP was undertaken using peer-reviewed journal articles. The use of peer-reviewed work only was considered crucial in providing some degree of quality assurance of the research evaluated. The search for papers was undertaken using academic databases that together cover all relevant journals. Each database was searched using keywords related to 'marine planning', 'marine spatial planning' and 'maritime spatial planning'. Wildcards were included to identify any additional papers that did not explicitly use these phrases. A total of 96 articles were identified. Each of these was then carefully read in order to identify 'claims of effective practice'. A claim of effective practice was identified as a statement specifying that a certain procedure, activity, or approach leads to effective MSP practice. Each claim was recorded, as was the type of evidence underpinning the claim. In order to maintain comparability within the evaluation, the evidence base was either categorised as being based upon primary data (where the claim was underpinned by the results of a survey) or based upon expert opinion (where the claim was underpinned by the experience, view or opinion of the authors of the article). The geographical scope of the review was international, although the publications were limited to those written in English.

\section{Results}

As might be expected, a meta-analysis is only as reliable as the papers identified and used within it. Of the 96 papers reviewed, only three used primary data sources as the main evidence base for their claims, with the remainder dependent upon opinion, experience, and personal judgement (collectively termed 'expert judgement'). Whilst expert judgement has its place in determining effective practice, primary data is particularly important in providing objective data from which the reader can formulate their own opinions. In papers that present expert judgement, the reader has little choice but to accept the interpretation of the author as there is no primary evidence to indicate the evidence base from which an author has developed their views.

As Table 1 shows, a total of 49 claims of effective practice were made in the papers reviewed. These were dominated by claims related to stakeholder involvement, which totalled 24 claims, $49 \%$ of the entire total. The remainder were fairly evenly spread across a range of areas of effective MSP practice, each receiving two to four claims. The results are presented in thematic groups in sections 3.1-3.5..

\begin{tabular}{|l|c|c|c|}
\cline { 3 - 4 } \multicolumn{2}{c|}{} & \multicolumn{2}{c|}{ Evidence for claim } \\
\hline Claim topic & Total claims & Expert judgement & Primary data \\
\hline Evidence base & 6 & 6 & 0 \\
\hline
\end{tabular}




\begin{tabular}{|l|c|c|c|}
\hline Stakeholder Involvement & 24 & 21 & 3 \\
\hline Objectives and goals & 3 & 3 & 0 \\
\hline Temporal scale & 2 & 2 & 0 \\
\hline Ecosystem based approach & 3 & 3 & 0 \\
\hline Spatial scale & 3 & 3 & 0 \\
\hline Economy & 1 & 1 & 0 \\
\hline Sectoral integration & 2 & 2 & 0 \\
\hline Legal framework & 3 & 3 & 0 \\
\hline Monitoring and adaptation & 2 & 2 & 0 \\
\hline TOTAL & $\mathbf{4 9}$ & $\mathbf{4 6}$ & $\mathbf{3}$ \\
\hline
\end{tabular}

Table 1. Summary of claim topics of effective practice identified from the systematic review of 96 MSP peer-reviewed publications.

\subsection{The evidence base for MSP should be the best available}

From the literature reviewed there were three explicit claims that the best available evidence (including local knowledge) underpins effective MSP, (Gilliland, 2008; Jay 2010). It was suggested that a 'stocktaking' approach should be undertaken, in which an in-depth analysis of the major actors, laws and institutions that influence MSP should be identified and considered (Oral, 2008). This should include consideration of all governance interactions with marine and adjoining coastal ecosystems, thus providing a strong baseline against which future change could be evaluated (Morrison, 2008). Where information is insufficient, the precautionary principle should be employed until the time where relevant information becomes available. It was claimed that the evidence base should be presented map-form that visually conveys relevant features of the marine environment, its use, and governance. Harris (2008) claims that a biodiversity map is instrumental to this process which would be combined with the spatial distribution of human activities and their governance to allow for integrated decisions.

\subsection{Stakeholder Participation is critical but must be informed}

There were a total of 24 claims of effective practice related to the role of stakeholder involvement in MSP including claims arising from the three papers that included primary evidence. Stakeholder involvement was therefore the most commonly cited effective practice claim within the literature reviewed. Claims included that stakeholder involvement should be considered intrinsic to MSP; it should offer a process that led to stakeholder empowerment; that participation should be early in the MSP process and then continue throughout the process; that the 'right' stakeholders should be engaged in MSP; and that stakeholder involvement should be used to obtain and harness local knowledge. It was acknowledged that this would require considerable effort and capacity building to ensure that stakeholders understand their role in MSP. Once stakeholders have been identified, continuous effort is required by a Marine Planner to build and maintain a rapport with stakeholders, not only to gain trust and to support the process, but to build commitment, a sense of shared responsibility and ownership (Pomeroy and Douvere, 2008). However, Ehler and Douvere (2006) warn that stakeholder engagement should inform and support, "but not to be the sole determinant of any MSP scheme". It is clear that given the multiple facets of stakeholder involvement in MSP, the challenges involved in delivering it effectively should not be underestimated. Similarly, whilst it is recognised that stakeholder participation is critical to a success of the MSP process, it was also clear that participation alone is not sufficicent to guarantee effective practice, as stakeholders need to be empowered to enable their full engagement, potentially through effective capacity building. 


\subsection{Sectoral and spatial integration is necessary}

Four claims related to the need for sectoral and spatial integration in effective MSP. First, it was claimed that in order to be effective, MSP should include all interested sectors, a claim that connects with much of the literature on coastal governance. As many sectors have their own sector-based plans, it was claimed that a key task of MSP is to identify synergies with other sectors of the economy and consider how mutual advantages could be identified. Activities in other sectors may have major implications for the marine sector, and vice versa, therefore an integrated approach was considered to be important. In particular, it was noted that MSP presents an opportunity to increase the financial benefits of marine industries, whilst providing protected areas for tourism and conservation. It is vitally important, however, that the MSP process is all inclusive and considers the entire spectrum of the economy, and not to show a preference to any single industry. The spatial fragmentation of governance is a significant risk in effective spatial planning of any sort, therefore making connections between existing sectoral plans and other spatial plans is an important strategy in effective MSP.

\subsection{MSP must be ecosystem-based at appropriate spatial and temporal scales}

It was claimed that MSP should be ecosystem-based, or at least be part of a wider ecosystem-based framework for marine administration (Crowder and Norse, 2008; Douvere, 2008; Gilliland and Laffoley, 2008; Maes, 2008). Effective MSP, it is claimed, is therefore working towards ecosystem integrity (Jay, 2010), which in turn has clear links to biodiversity conservation, and the provision of ecosystem services. Therefore from the papers reviewed, there were clear claims that MSP should be based upon the ecosystem approach and take into account the ecosystem services provided by marine ecological habitats and species. It was claimed that MSP requires clear and measurable goals and objectives. Goals relate to the aspirations of the entire process (such as to increase offshore wind energy by $20 \%$ ) whereas objectives are the mechanisms through which the goals are achieved (such as dedicated wind farm development zones). It was claimed that the extent to which both goals and objectives are achieved should be quantifiably measurable to allow for effective monitoring and adaptation. It was claimed that MSP goals and objectives should be attached to tightly defined timescales. A specific spatial scale at which MSP should be implemented was not suggested in the papers reviewed, yet several suggest that the spatial scale should be 'appropriate' (Kidd, et al., 2003; Douvere, et al., 2009; Yorio, 2009;). It was suggested that emphasis should be put on producing realistic plans using appropriate scales to compliment the prevailing physical and ecological processes.

\subsection{The implementation of MSP should be legally binding and monitored}

It was noted in the review that unlike terrestrial planning systems, marine planning systems are currently characterised by multiple governance bodies, a lack of clear rules and regulations, and an unclear process to balance economic development and environmental protection. As MSP evolves and matures, it was claimed that the laws surrounding marine protection and development should 'have teeth' and create an enforceable and binding mechanism (Morrison, et al., 2008). The need for monitoring of the implementation of MSP and subsequent adaptation of approaches and actions was claimed to be a necessary prerequisite of effective practice. Whilst monitoring and adaptation are typical components of most self-aware management approaches, the relative infancy of MSP in most of the world makes such an approach particularly important for MSP. It is noted by Ehler (2008) that the real value of the monitoring and evaluation processes is the level of acknowledgement the findings receive, in particular how they feed back to improve future management decisions. It was also claimed in the review that monitoring should not just be of the outcomes but also of the MSP working process itself. 


\section{Synthesis of effective practice}

From the review of 96 peer-reviewed papers focused on MSP, it has been possible to identify a range of claims of effective practice in MSP. A total of 49 claims were identified, ranging across 10 topic areas, although almost half of the claims related to the single topic of stakeholder involvement. In the analysis of the claims (presented in section 3), the categories were rationalised in order to remove repetition and overlap, however, when considered separately, it yields the following claims of effective practice:

In order for MSP to be effective...

- the evidence base for MSP should be the best available;

- $\quad$ stakeholder participation must be effective;

- stakeholder participation must be well informed;

- integration with other sector-specific plans is necessary;

- integration with other spatial plans is necessary;

- it must adopt an ecosystem-based approach;

- it must be undertaken at an appropriate spatial and temporal scale;

- $\quad$ its implementation should be legally binding; and

- its implementation should be monitored.

In considering these claims, it is important acknowledge that with the exception of three papers that included primary data, the vast majority of the evidence base for the effective practice claims outlined above was derived from expert judgement. To some extent, this may be a reflection of the limited application of MSP, creating very limited opportunities for primary data informed evaluation of practice. The claims of effective practice therefore perhaps more closely reflect the consensus view of what is thought will deliver effective MSP rather than what has delivered effective MSP. It should also be noted that the relative lack of evidence of effective practice in many areas of MSP operation does not imply that practice is ineffective, it simply implies that the evidence is, at present, not available. There are, therefore, significant research gaps related to MSP operation.

\section{Conclusion}

The key conclusion from this research process relates to the very limited amount of peerreviewed research that includes primary data about MSP projects and approaches. As such, there is limited evidence as to what has actually delivered effective MSP practice as opposed to what is anticipated will deliver effective MSP. However, despite this, there was considerable agreement in the existing research literature, that in order to be effective, MSP should draw from the best available evidence base, involve informed stakeholders, integrate with existing sectoral and spatial plans, adopt an ecosystem-based approach at appropriate spatial and temporal scales, be implemented through a legally binding framework, and be subject to rigorous monitoring. These conclusions may vary over time as greater levels of primary research is carried out into MSP programmes which creates a stronger evidence base for effective practice.

\section{References}

Crowder, L. and Norse, E. 2008. Essential ecological insights for marine ecosystem-based management and marine spatial planning. Marine Policy 32(5): 772-778.

Douvere, F., Maes, F., Vanhulle. A, and Schrijvers, J. 2007. The role of marine spatial planning in sea use management: The Belgian case. Marine Policy. 31:182-191. 
Douvere, F and Ehler, C.N. 2009. New perspectives on sea use management: Initial findings from European experience with marine spatial planning. Journal of Environmental Management 90:77-88.

European Commission. 2008. Roadmap for maritime spatial planning: Achieving common principles in the EU. EC, Brussels.

European Commission. 2009. Preparatory action on maritime spatial planning in the NE Atlantic / North Sea / Channel area. EC, Brussels.

Ehler, C. 2008. Conclusions: Benefits, lessons learned, and future challenges of marine spatial planning. Marine Policy 32(5): 840-843.

Ehler, C. and Douvere, F. 2006. Visions for a sea change: report of the First International Workshop on Marine Spatial Planning Intergovernmental Oceanographic Commission and the Man and the Biosphere Programme, UNESCO Paris.

Gilliland, P. M. and Laffoley, D. 2008. Key elements and steps in the process of developing ecosystem-based marine spatial planning. Marine Policy 32(5): 787-796.

Jay, S. 2009. Planners to the rescue: Spatial planning facilitating the development of offshore wind energy. Marine Pollution Bulletin. 60:493-499.

Kidd, S., Massey, D. and Davies, H. 2003. The ESDP and integrated coastal zone management - Implications for the integrated management of the Irish Sea. Town Planning Review. 74: 97-120.

Maes, F. 2008. The international legal framework for marine spatial planning. Marine Policy $32: 797-810$.

Morrison, K. and Snow-Cotter, S. 2008. Toward More Integrated Ocean Governance in Massachusetts: A Progress Report. Coastal Management 36:412-430.

Pomeroy, R. and Douvere, F. 2008. The engagement of stakeholders in the marine spatial planning process. Marine Policy 32:816-822.

Stelzenmüller, V., Ellis, J. R. and Rogers, S.I. 2008. Towards a spatially explicit risk assessment for marine management: Assessing the vulnerability of fish to aggregate extraction. Biological Conservation 143: 230-238.

Yorio, P. 2009. Marine protected areas, spatial scales, and governance: implications for the conservation of breeding seabirds. Conservation Letters 2:171-178. 